\title{
Virtual Fossils: a New Resource for Science Communication in Paleontology
}

\author{
Imran A. Rahman • Keith Adcock • \\ Russell J. Garwood
}

Published online: 10 November 2012

(C) Springer Science+Business Media New York 2012

\begin{abstract}
Computer-aided 3-D reconstruction of fossils, or virtual paleontology, is an increasingly common and powerful technique. It is now regularly used for research in paleontology, yet to date has impacted little on public outreach and science communication; however, it is ideally suited for these purposes, being increasingly cheap and available, dynamic and exciting, and applicable to a range of topics. Here, we provide an introduction to the field, and a case study of its use for a public engagement event. The steps involved in creating such an educational resource are outlined, and include computed tomography scanning, digital visualization, and 3-D printing of fossils. We emphasize the value of virtual fossils for science communication; they allow for diverse learning styles in a variety of topics. In the future, we hope that virtual paleontology will become a mainstay of communicating the history of life, thereby promoting accurate understanding of evolution.
\end{abstract}

Electronic supplementary material The online version of this article (doi:10.1007/s12052-012-0458-2) contains supplementary material, which is available to authorized users.

I. A. Rahman $(\bowtie)$

School of Earth Sciences, University of Bristol,

Wills Memorial Building, Queen's Road,

Bristol BS8 1RJ, UK

e-mail: Imran.Rahman@bristol.ac.uk

K. Adcock

Jewellery Industry Innovation Centre,

Birmingham City University,

Vittoria Street, Hockley,

Birmingham B1 3PA, UK

\section{R. J. Garwood}

Schools of Materials and Earth, Atmospheric and Environmental Sciences, University of Manchester, Oxford Road,

Manchester M13 9PL, UK
Keywords Paleontology · Public engagement · Virtual fossils $\cdot$ Computed tomography $\cdot 3$-D printing $\cdot$ VAXML

\section{Introduction}

Fossils provide us with the only direct evidence of prehistoric life. Without them we would know next to nothing about captivating extinct groups such as non-avian dinosaurs, trilobites, and woolly mammoths. Nevertheless, fossils can frustrate as well as enlighten; fossilization typically flattens dead creatures, leaving 2-D (two-dimensional) specimens that are difficult to understand as real, 3-D (threedimensional) organisms. Under unusual circumstances, however, fossils can be three-dimensionally preserved; for example, if a mineralized concretion formed around the remains of an organism soon after burial (Fig. 1). Such material is critical, providing scientists with an unparalleled opportunity to reconstruct the biology and evolution of extinct creatures. Owing to their spectacular appearance, exceptional three-dimensionally preserved fossils are also ideal tools for educating non-specialists in paleontology. However, the most outstanding specimens are extremely rare and often very fragile; they are therefore generally not accessible to the public, despite their strong potential interest.

The aim of this article is to propose a resource that will allow anyone, not just specialists, to interact with rare and precious fossils specimens. While the display of original fossils in museums and institutions is, and should remain, a cornerstone of paleontological outreach (Erwin and Zeigler 1997; Eldredge 2005; Lieberman and Kaesler 2010), with recent technological advances and their widespread application, it is now practical to produce digital and physical 3-D models of fossils for the purposes of education and communication. Such reconstructions represent an innovative new means of engaging the public with 


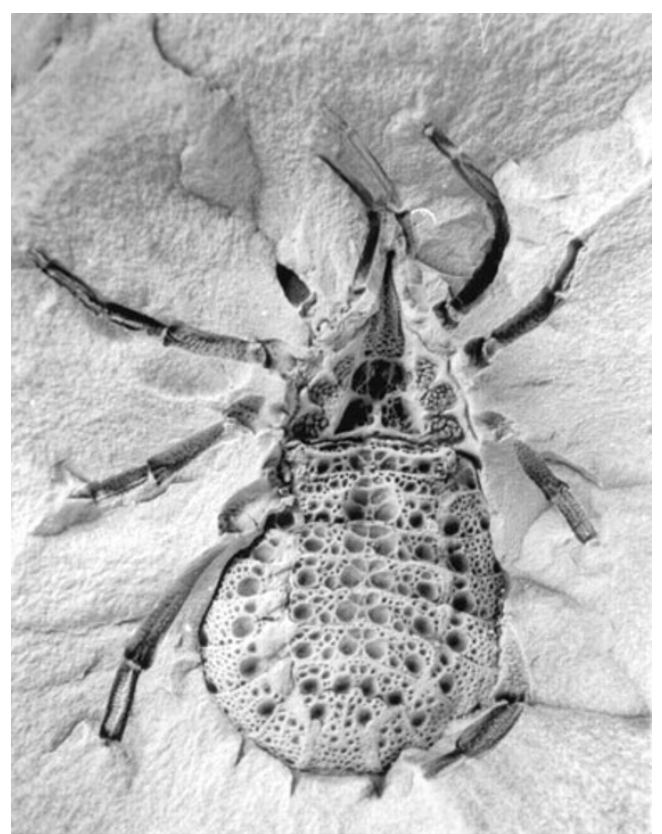

Fig. 1 Three-dimensionally preserved fossil of the trigonotarbid arachnid Eophrynus prestvicii, hosted within an iron carbonate concretion. The specimen is from the Late Carboniferous Coal Measures ( 311 million years ago) of the UK, and is housed in the Lapworth Museum of Geology, University of Birmingham, UK (specimen number: BU699). Fossil is $\sim 30 \mathrm{~mm}$ in length

paleontology. This paper outlines the state-of-the-art techniques required to produce these "virtual fossils", and presents a case study of their use in science communication. It is hoped that this will serve as a useful guide for those seeking to implement this approach in their own engagement and teaching activities.

\section{Approaches for Reconstructing Virtual Fossils}

Virtual paleontology - computer-aided visualization of fossils - is becoming increasingly important in paleontological research. The 3-D reconstruction of a sample's internal and external morphology can reveal previously unknown details that have important evolutionary implications (e.g., Dierick et al. 2007; Rowe et al. 2008; Selden et al. 2008; Garwood et al. 2009; Dunlop et al. 2012; Zamora et al. 2012). However, this approach has yet to be widely used for public engagement, in part because the techniques involved are thought of as expensive and inaccessible. This is no longer the case; modern methods for digitally capturing fossils in three dimensions are both affordable and rapid (although data processing can be time-consuming). Furthermore, they allow data routinely acquired for scientific research to be employed at little extra cost for outreach activities.

For many years, paleontologists have ground or sawn through fossils (often brachiopods) in order to see their internal anatomy in cross-section (e.g., Sollas 1904; MuirWood 1934; Schemm-Gregory and Sutton 2010). This approach can be informative, but is extremely laborious and results in the complete destruction of the fossil. With modern scanning technology and the right specimen, slices almost as good as physically revealed surfaces can now be imaged without damaging the fossil. The most widely used scanning method in paleontology is computed tomography (CT) (Anderson et al. 2003; Abel et al. 2012). This technique is applicable to a wide range of preservation types and specimen sizes, and is perhaps the most effective means of generating data that can be used to reconstruct a virtual fossil.

\section{Computed Tomography}

CT is an X-ray-based scanning technology used widely in hospitals for diagnosing internal injuries and diseases; the flexibility of the technique has led to numerous other applications. CT scans are relatively quick to acquire, cheap, and non-destructive. They produce cross-section images which map the internal and external morphology of an object; CT is therefore ideal for imaging irreplaceable fossil specimens. Medical scanners use low-energy X-rays and short exposure times to minimize radiation doses for patients, but nonmedical CT has no such restrictions; systems can be optimized to penetrate rock and generate high-resolution images (resolving details down to one hundredth of a millimeter or less). During a CT scan of a fossil, X-rays are passed through the specimen while it rotates around $180^{\circ}$ or $360^{\circ}$. The extent to which the X-rays are attenuated (absorbed or scattered) by the sample is mapped in three dimensions using a computer algorithm. X-ray attenuation roughly equates to density, so it can distinguish between different mineral phases - for example, fossil and rock-as long as they attenuate X-rays differently. Scan results are most easily thought of as a dataset of hundreds or even thousands of slice images, each revealing fine morphological details (Fig. 2). In paleontological studies, medical CT scanners have been useful for imaging large vertebrate fossils (e.g., dinosaur skulls), but the miniaturized version of $\mathrm{CT}$, termed micro-CT, is the optimum technique for studying smaller vertebrate, plant, and invertebrate fossils.

High-end CT scanners are available at a number of universities and research institutions across the globe. Specialist facilities where CT has been successfully used to study fossils include: the Centre for X-ray Tomography at Ghent University, Belgium; the Henry Moseley X-ray Imaging Facility at the University of Manchester, UK; the Imaging and Analysis Centre at the Natural History Museum, London, UK; the $\mu$-VIS Centre at Southampton University, UK; and the High-Resolution X-ray Computed Tomography Facility at the University of Texas at Austin, USA. Obtaining time on machines in such centers, which 
Fig. 2 CT slice showing a cross-section through the trigonotarbid arachnid E. prestvicii. The fossil is preserved as a void in an iron carbonate concretion. Concretion is $\sim 75 \mathrm{~mm}$ in width

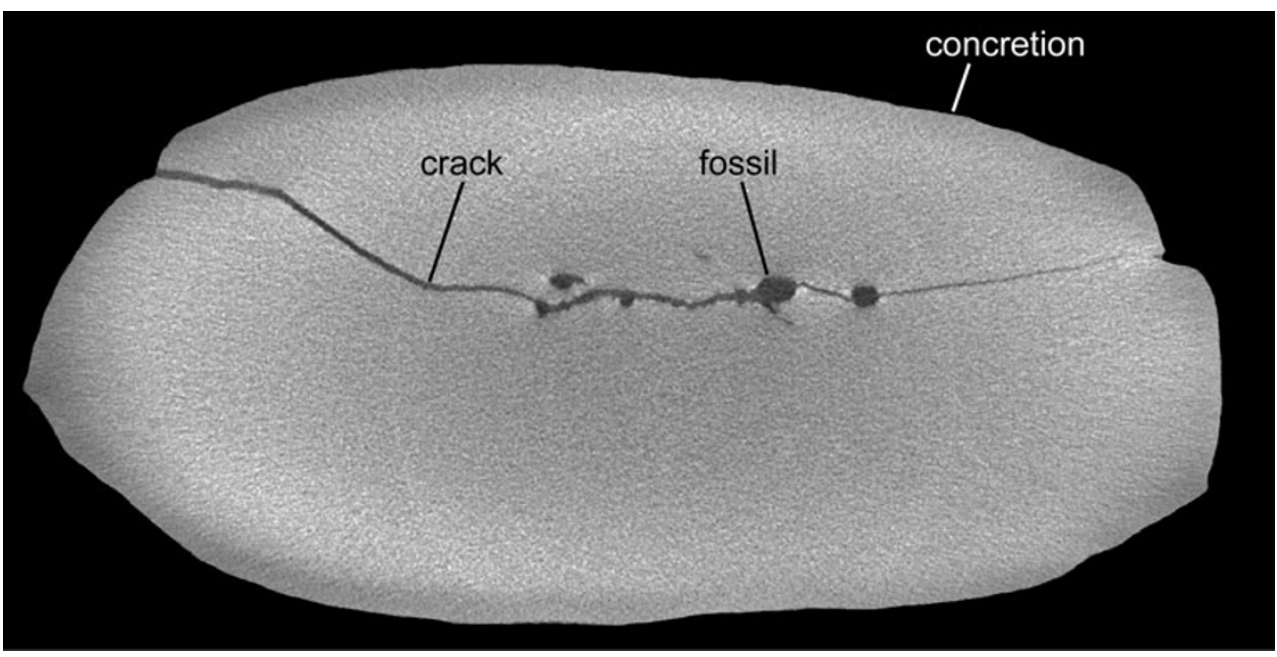

typically charge subsidized rates for academic and educational projects, is feasible for many potential users.

\section{Computer Visualization}

Tomographic slices can be studied directly - in fact, this approach has a rich history in paleontology — but, with the aid of modern computational techniques, are most usefully employed for producing 3-D models. Historically, fossil workers visualized slice datasets by constructing physical models (e.g., Sollas 1904; Sollas and Sollas 1914; Jefferies and Lewis 1978). These reconstructions proved difficult to dissect, and maintaining articulation of non-contiguous parts was impossible. In the last two decades, the advent of powerful yet relatively cheap computers has revolutionized the field, ushering in the birth of virtual paleontology. Digital visualization, which allows users to study models that can be viewed in any orientation, colored, virtually dissected, and made locally translucent, is now becoming widespread (Sutton 2008; Garwood et al. 2010). There are two main approaches for creating a virtual fossil from CT data. The first is volume rendering, in which 2-D pixels in slice images are treated as voxels in a 3-D array ( 3-D pixels). Models are visualized by projecting virtual beams of light through these volumes, and the light rays are affected by the voxels based on user-defined rules. This approach is quick to set up, but is often slow to render, and can produce noisy reconstructions. The second, and slightly more common, approach is to model surfaces wrapped over the voxels of a dataset. To facilitate this, the voxels are converted into binary (thresholded) images, again on the basis of user-defined rules. Resulting surfaces are then modeled with a triangle mesh (Sutton et al. 2001). Using both these approaches, datasets can be cleaned and edited, if needed, or visualized straight off a scanner. Virtual specimens are normally presented as 2-D images (Fig. 3) or prerendered animations (Online Resource 1).
A large number of software packages are available that can be used to produce 3-D digital visualizations from slice images. Most such programs require expensive user licenses; SPIERS, in contrast, is free software that runs well on low-powered computers, and is specifically designed for paleontological data. In addition, SPIERS utilizes a standardized interchange format, VAXML, meaning it can be used to view models created using different programs (Sutton et al. 2012). It is envisaged that an online database of VAXML files will be established in the near future, allowing scientists and the public alike to view and interact with digital models of fossils for free. SPIERS is suggested as a good starting point for anyone new to virtual paleontology - the program and comprehensive, up-to-date documentation can be downloaded from: www.spiers-software.org.

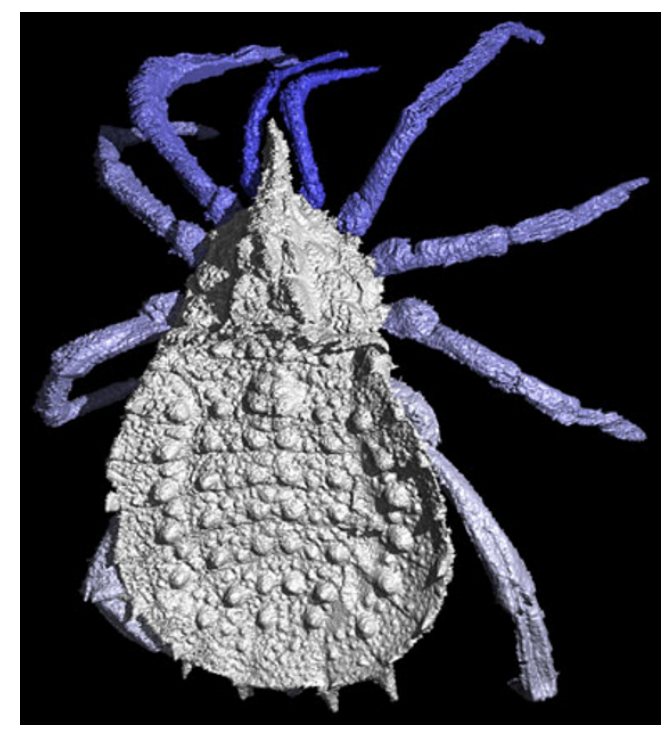

Fig. 3 Computer reconstruction of the trigonotarbid arachnid E. prestvicii, with the surrounding rock virtually removed. Fossil is $\sim 30 \mathrm{~mm}$ in length 


\section{3-D Printing}

An additional advantage of this approach to paleontology is that valuable fossils can be printed in 3-D from digital models (Fig. 4). This technique has, in recent years, become cheaper and more accurate. Prints are usually created with polymers, or sometimes metals, that are hardened or fused, layer-bylayer, to create a physical representation of the virtual specimen. This process allows fossils to be presented in museums as tactile exhibits, which can be displayed along with the original fossil and high-quality images/animations of the computer reconstruction. Using these techniques, tiny specimens can be enlarged until micrometer-scale features can be seen with the naked eye, and even the most fragile fossils can be safely handled by non-specialists. Furthermore, such objects potentially allow blind and visually impaired people to engage with fossils through touch (Teshima et al. 2010), an important step towards accessibility in a traditionally highly visual field.

3-D printers are often found in manufacturing, industrial design, research, and increasingly arts institutions. Moreover, several companies offer online 3-D printing services; customers upload their digital model in the correct format to the company's website, and it is then printed in the desired color/material and shipped back to them. Examples of these companies include: i.materialise, Sculpteo, and Shapeways. Alternatively, selfassembly 3-D printer kits are now available at reasonable prices, and these may be purchased for desktop use.

\section{Case Study: Using Virtual Fossils for Public Engagement}

To test the utility of virtual fossils for science communication, a pilot resource consisting of digital reconstructions

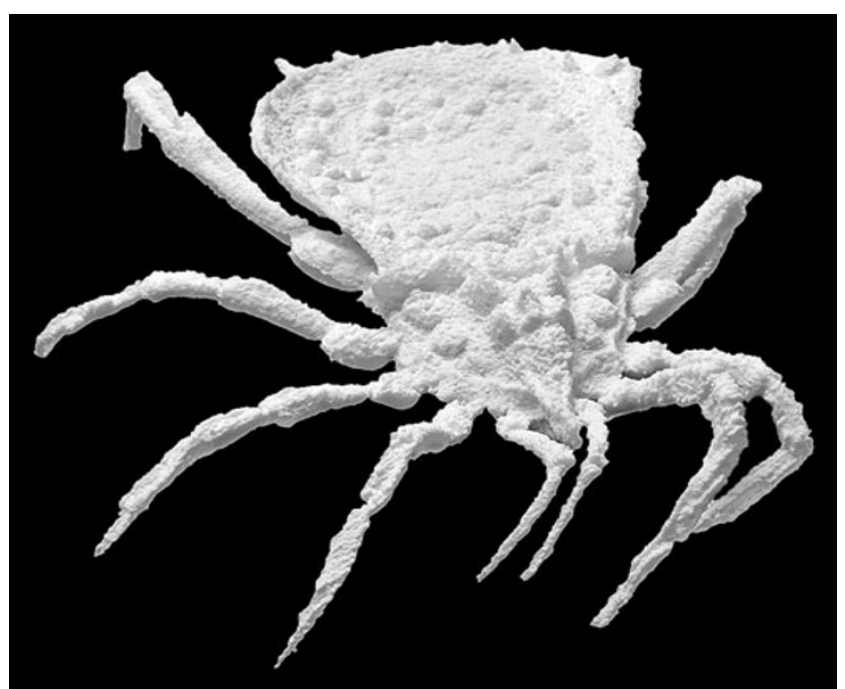

Fig. 4 3-D printed model of the trigonotarbid arachnid E. prestvicii. Object is $\sim 140 \mathrm{~mm}$ in length and 3-D printed models of fossils was created and exhibited in early 2012 (Figs. 5 and 6). This work was financed by the Natural Environment Research Council (NERC, a UK funding body that supports research, training, and knowledge exchange in the environmental sciences) through a new scheme for developing resources to promote public engagement with research (http://www.nerc.ac.uk/about/work/ engagement/scheme.asp). The website of the National Coordinating Centre for Public Engagement provides a list of other relevant funding opportunities for UK researchers and teachers (http://www.publicengagement.ac.uk/how/funding).

\section{Creating the Resource}

The first step was to identify suitable fossils for inclusion in the resource. Representatives from several different invertebrate groups (arthropods, brachiopods, echinoderms, and mollusks) were incorporated so that the resource could be used to provide a broad education in some lesser-known extinct animals. In addition, fossils were selected that met the following three criteria, which were deemed critical to the success of the project: (1) 3-D preservation. Flattened fossils can be difficult to CT scan (Abel et al. 2012), and visualizing them in 3-D will generally not provide any significant information that could not be obtained by

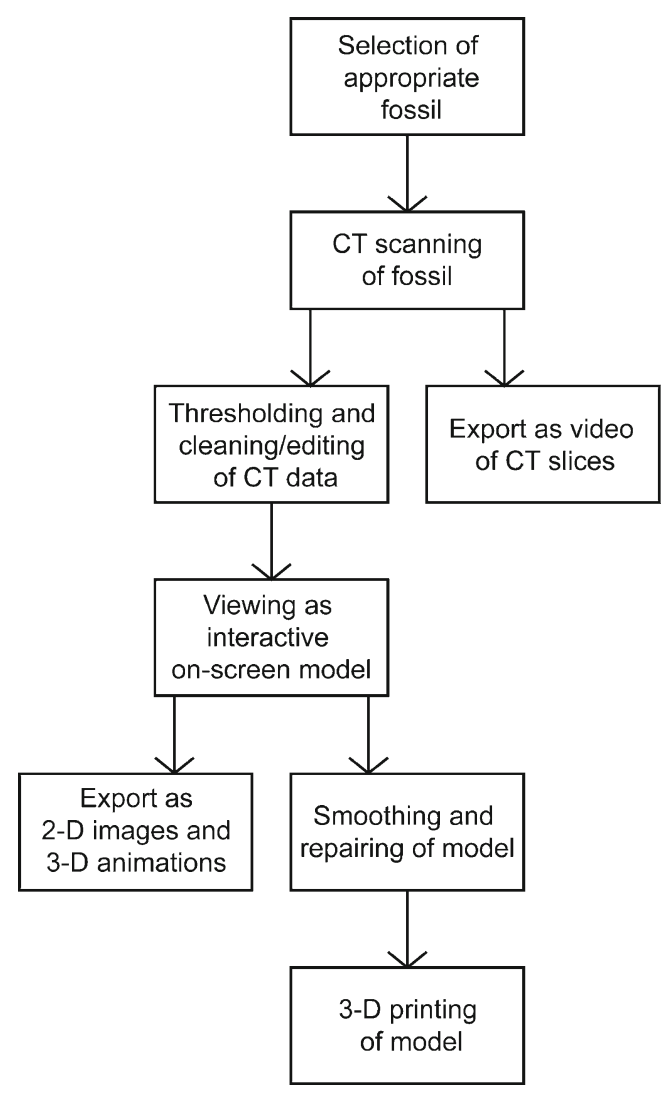

Fig. 5 Workflow for creating a virtual paleontology resource for public engagement 
Fig. 6 Photograph of the virtual paleontology resource during its display at the Lapworth Museum of Geology, University of Birmingham, UK

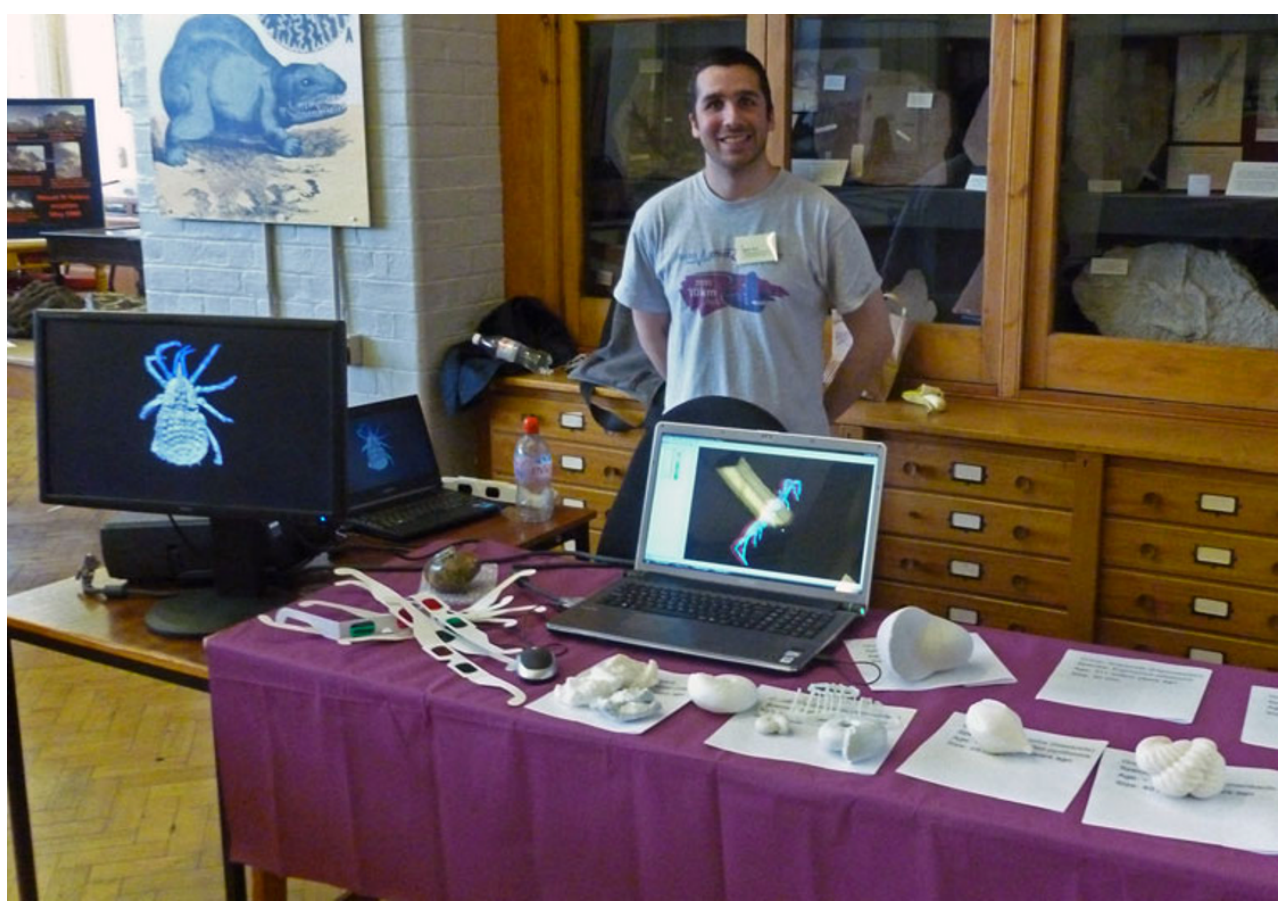

viewing them under a microscope. Three-dimensionally preserved fossils are much better suited for a resource of this kind. (2) Preservation of fine morphological details. Poorly preserved specimens, even if 3-D, typically lack many important anatomical details. In contrast, digital reconstructions of well-preserved fossils allow us to visualize and magnify previously hidden structures, and are therefore ideal tools for engagement. (3) Appropriate size, density, and contrast for CT scanning. Most lab-based CT scanners are incapable of imaging fossils larger than approximately $250 \mathrm{~mm}$ (Abel et al. 2012). Moreover, samples composed of dense materials (e.g., iron-rich minerals) can be difficult to penetrate using standard X-ray energies. Finally, fossils hosted in a compositionally similar matrix are difficult to image using CT because they lack substantial density contrast. Isolated fossils or those preserved as voids in rock are the easiest to image using CT, and do not usually require extensive data processing.

The selected fossils were scanned at high-resolutions (less than one tenth of a millimeter) on one of three different CT scanners, depending on availability: (1) a SkyScan 1172 in the School of Dentistry at the University of Birmingham, UK; (2) a Phoenix v|tome $\mid \mathrm{x} s$ in the Department of Materials, Imperial College London, UK; or (3) a Nikon X-Tek HMX-ST 225 in the Imaging and Analysis Centre at the Natural History Museum, London, UK. CT datasets were reconstructed as virtual fossils using the free SPIERS software suite (Sutton et al. 2012). Due to the careful selection criteria exercised prior to scanning (see above), limited manual cleaning/editing in SPIERSedit was required to produce an informative computer reconstruction; however, some post-processing was necessary in order to produce files suitable for 3-D printing. Digital models were exported in STL format and were smoothed and simplified in SPIERSview or the open source software MeshLab (http:// meshlab.sourceforge.net). Meshes were then repaired (holes were closed, and self-intersections and parallel planes removed) using the free program netfabb Studio Basic (http:// netfabb.com/basic.php). The resulting digital reconstructions, in VAXML format, are included in Online Resource 2.

3-D printing was undertaken at the Jewellery and Industry Innovation Centre, Birmingham City University, UK on an Objet Eden350. This system prints by jetting photo-polymeric resin in horizontal layers around one hundredth of a millimeter thick, which are cured by a pair of UV lamps as they are printed. Support material surrounds the build in each layer, allowing overhanging structures to be printed. This supporting material is similar to the build material, but contains an inhibitor that prevents it from hardening, leaving it as a jelly-like substance. The support and build were printed at the same time, with different jet types switching on and off, in turn. After printing, the soft support material was manually removed from the build, giving a clean, uniform finish. The final models are highly detailed and accurate replicas of the original fossils.

\section{Exhibiting the Resource}

The materials described above were displayed at the Lapworth Museum of Geology, University of Birmingham, UK, during the annual University Community Day in June 2012 (Fig. 6). This is a 1-day event where local residents are 
invited to attend various free activities across the university; exhibiting the resource as part of this established event was thought to be the most effective way to ensure engagement with a large number of members of the general public. During the exhibit, computer reconstructions were run on a laptop and projected onto a portable screen in red/green anaglyph stereo. Visitors were able to interact with the computer reconstructions using SPIERS, and view the models projected on screen with supplied 3-D glasses. 3-D prints of fossils were displayed next to the laptop along with brief details (taxonomic group, species name, age, and size of the original fossil), and visitors were encouraged to handle these items. A second laptop connected to an external monitor was used to show a video describing the virtual paleontology approach (Online Resource 3). Two researchers (Imran Rahman and Russell Garwood) were on hand throughout the day to supervise the exhibit, talk to the public, and answer any questions.

Visitors consisted primarily of young children and their parents; different approaches were required to successfully communicate our findings to the two distinct age groups. The children tended to be most interested in the 3-D glasses and prints of fossils; engagement with this group was achieved by facilitating direct interaction with these materials, while at the same time pointing out specific features visible in the virtual fossils that have important implications for the biology of the extinct creatures. Taking the trignotarbid arachnid Eophrynus prestvicii (Figs. 1, 2, 3, and 4) as an example, we emphasized the presence of long limbs and a heavily armored body (features which are clear in both the digital and 3-D printed models), adaptations to an active, predatory mode of life. Older children were asked to identify similarities between this fossil and the trilobite on display. The shared features, contrasted with the other fossils, served as an introduction to the arthropods, and to biological classification in general. The parents were more broadly interested in the resource, and asked questions about the technologies used to create the models, as well as the fossils themselves. Thus, to enhance engagement with this audience, longer and more detailed conversations were undertaken; the methods were described at length, and also the transformative effect of these approaches on modern paleontology, with examples of their application to active research questions.

\section{Discussion}

Engagement events such as the case study outlined herein provide incredibly valuable opportunities to advance the public understanding of science. Current evidence, while inconclusive, suggests that free-choice science learning experiences (e.g., museums, media, and community organizations) make a greater contribution to public understanding of science than formal school education (Falk and Dierking 2010). This is particularly important in subjects associated with evolution, which can be more contentious and less widely accepted than many scientific topics. This is especially true in regions where religious fundamentalism is coupled with the politicization of science, for example in the Middle East (Burton 2010) and the US (Miller et al. 2006), where it is impacting science education (Garwood 2012).

Fossils are ideal for these free-choice learning experiences. Extinct species have long fascinated both children and adults, as attested to by their impact on popular culture (see, for example, the Jurassic Park movies). The fossilized remains of such creatures, and their geological context, provide direct evidence for evolution. A lack of understanding of the fossil record has been reported as an obstacle to biology education (Nelson 2008). 3-D visualization and printing of virtual fossils adds a technological slant to further entice visitors, while demonstrating the breadth of modern paleontology (and countering stereotypes of paleontology as a dated science, and the scientists in the field as traditional and resistant to change). Furthermore, in contrast to many real specimens, these fossils can be handled, making what is traditionally a visual discipline into one well suited to tactile/kinesthetic learning, particularly valuable for young children (Dunn and Dunn 1992). For older children and adults, careful choice of fossils allows the introduction of complex ideas such as biological classification and the hierarchical organization of life, in addition to the evolutionary causes underlying these concepts. Inductive learning experiences such as these are valuable, and contrast with the often deductive teaching methods of traditional science education (Prince and Felder 2006).

\section{Conclusions}

Virtual paleontology is a valuable technique for studying the history of life, and has enormous potential as a public outreach resource-it represents a unique way for people to actively engage with fossils to which they would usually have no access. The inherent interactivity of virtual fossils makes them effective tools for engaging with the public, particularly young people, and the tactile nature of displays is important for reaching non-visual learners. By making use of state-of-the-art technologies such as CT scanning and 3-D printing, virtual paleontology also appeals to adults who might not normally be interested in fossils, and this represents an excellent route to educating this group in natural history and evolution - topics which remain poorly understood by many. Public engagement work of this sort is cost efficient, as it makes use of methods and data regularly used in scientific research, as well as freely available software 
and computer models. This makes virtual paleontology a potential means to provide free-choice learning on a large scale, and one which is ideal for museums and other institutions with limited public funding. The choice of fossils allows a broad range of topics to be addressed, including complex concepts, in a supportive environment. In the future, virtual paleontology is likely to become an even betterestablished and more accessible technique. We foresee the creation of freely available teaching resources to use with this free software, encouraging the technique's use in educational environments. The marriage of computer science and the study of past life has created an incredibly exciting field-one which should not be limited to an academic sphere. We believe that with concerted efforts to communicate this work, virtual paleontology can increase the public understanding of evolution, and provide a valuable addition to the biological educator's armory.

Acknowledgments We thank Poppy Leeder, Myfanwy Johns, Frank Cooper, and Jon Clatworthy for their help and support throughout the project. We also thank Richard Hamilton and Michelle Holder for assistance with CT scanning. We are grateful to two anonymous reviewers for their comments. This work was funded by the Natural Environment Research Council (NERC) Public Engagement with Research Resource Development Funding Scheme. Imran Rahman was supported by a NERC Postdoctoral Research Fellowship (grant number NE/H015817/1). Russell Garwood was supported by an 1851 Research Fellowship.

\section{References}

Abel RL, Laurini CR, Richter M. A palaeobiologist's guide to 'virtual' micro-CT preparation. Palaeontol Electron. 2012;15(2):6T.

Anderson J, Caroll RL, Rowe TB. New information on Lethiscus stocki (Tetrapoda: Lepospondyli: Aistopoda) from highresolution computed tomography and a phylogenetic analysis of Aistopoda. Can J Earth Sci. 2003;40(8):1071-83.

Burton EK. Evolution and creationism in Middle Eastern education: a new perspective. Evolution. 2010;65(1):301-4.

Dierick M, Cnudde V, Massachaele B, Vlassenbroeck J, Van Hoorebeke L, Jacobs P. Micro-CT of fossils preserved in amber. Nucl Instrum Meth A. 2007;580(1):641-3.

Dunlop JA, Wirth S, Penney D, McNeil A, Bradley RS, Wither PJ, Preziosi RF. A minute fossil phoretic mite recovered by phasecontrast X-ray computed tomography. Biol Lett. 2012;8(3):457-60.

Dunn R, Dunn K. Teaching elementary students through their individual learning styles: practical approaches for grades 3-6. Boston: Allyn and Bacon; 1992.

Eldredge N. Darwin: discovering the tree of life. New York: W.W. Norton; 2005.
Erwin E, Zeigler W. Paleontology in museums and institutes in the 21st century. Kleine Senckenberg-Reihe. 1997;25:69-75.

Falk JH, Dierking LD. The 95 percent solution. Am Sci. 2010;98 (6):486-93.

Garwood RJ. Reach out to defend evolution. Nature. 2012;485 (7398):281.

Garwood RJ, Dunlop JA, Sutton MD. High-fidelity X-ray microtomography reconstruction of siderite-hosted Carboniferous arachnids. Biol Lett. 2009;5(6):841-4.

Garwood RJ, Rahman IA, Sutton MD. From clergymen to computers - the advent of virtual palaeontology. Geol Today. 2010;26 (3):96-100.

Jefferies RPS, Lewis DN. The English Silurian fossil Placocystites forbesianus and the ancestry of the vertebrates. Phil Trans R Soc B. 1978;282(990):205-323.

Lieberman BS, Kaesler RA. Prehistoric life: evolution and the fossil record. Oxford: Wiley-Blackwell; 2010.

Miller JD, Scott EC, Okamoto S. Public acceptance of evolution. Science. 2006;313(5788):765-6.

Muir-Wood HM. On the internal structure of some Mesozoic Brachiopoda. Phil Trans R Soc B. 1934;223:511-67.

Nelson CE. Teaching evolution (and all of biology) more effectively: strategies for engagement, critical reasoning, and confronting misconceptions. Integr Comp Biol. 2008;48(2):213-25.

Prince MJ, Felder R. Inductive teaching and learning methods: definitions, comparisons, and research bases. J Eng Educ. 2006;95 (2):123-38.

Rowe T, Rich TH, Vickers-Rich P, Springer M, Woodburne MO. The oldest platypus and its bearing on divergence timing of the platypus and echidna clades. Proc Natl Acad Sci U S A. 2008;105 (4):1238-42.

Schemm-Gregory M, Sutton M. First report of brachiopod-brachiopod endoparasitism. Lethaia. 2010;43(1):111-5.

Selden PA, Shear WA, Sutton MD. Fossil evidence for the origin of spider spinnerets and a proposed arachnid order. Proc Natl Acad Sci U S A. 2008;105(52):20781-5.

Sollas WJ. A method for the investigation of fossils by serial sections. Phil Trans R Soc B. 1904;196:259-65.

Sollas IBJ, Sollas WJ. A study of the skull of a dicynodon by means of serial sections. Phil Trans R Soc B. 1914;204:201-25.

Sutton MD. Tomographic techniques for the study of exceptionally preserved fossils. Proc R Soc B. 2008;275(1643):1587-93.

Sutton MD, Briggs DEG, Siveter DJ, Siveter DJ. Methodologies for the visualization and reconstruction of three-dimensional fossils from the Silurian Herefordshire Lagerstätte. Palaeontol Electron. 2001;4(1):2A.

Sutton MD, Garwood RJ, Siveter DJ, Siveter DJ. SPIERS and VAXML: a software toolkit for tomographic visualisation and a format for virtual specimen interchange. Palaeontol Electron. 2012;15(2):5T.

Teshima Y, Matsuoka A, Fujiyoshi M, Ikegami Y, Kaneko T, Oouchi S, Watanabe Y, Yamazawa K. Enlarged skeleton models of plankton for tactile teaching. Lect Notes Comput Sci. 2010;6180:523-6.

Zamora S, Rahman IA, Smith AB. Plated Cambrian bilaterians reveal the earliest stages of echinoderm evolution. PLoS One. 2012;7(6): e38296. 